Original

\title{
Proposal of an experimental data and image transmission system and its possible application for remote monitoring smart grids
}

\author{
Ibrahim Develi $^{\mathrm{a}, *}$, Yasin Kabalci ${ }^{\mathrm{b}}$ \\ ${ }^{a}$ Department of Electrical \& Electronics Engineering, Faculty of Engineering, Erciyes University, 38039, Kayseri, Turkey \\ ${ }^{\mathrm{b}}$ Department of Electronics and Automation, Nigde Vocational College of Technical Sciences, Nigde University, 51200, Nigde, Turkey
}

Received 14 July 2015; accepted 27 March 2017

Available online 27 May 2017

\begin{abstract}
This paper investigates the bit error rate (BER) and the peak signal-to-noise ratio (PSNR) performances of quasi-cyclic low-density parity-check (QC-LDPC) coded orthogonal frequency-division multiplexing (OFDM) systems over an actual power line communication (PLC) channel that are acquired by performing very long-term experimental measurements from the grid. The examined system is tested by changing system parameters such as code length, iteration number, coding rate and message type in detail. The results of this study show that the QC-LDPC coded OFDM system can be a possible solution for communication and remote monitoring purposes in smart grids.

(C) 2017 Universidad Nacional Autónoma de México, Centro de Ciencias Aplicadas y Desarrollo Tecnológico. This is an open access article under the CC BY-NC-ND license (http://creativecommons.org/licenses/by-nc-nd/4.0/).
\end{abstract}

Keywords: Smart grids; Remote monitoring; Power line communication (PLC); Quasi-cyclic low-density parity-check (QC-LDPC) codes; Orthogonal frequencydivision multiplexing (OFDM)

\section{Introduction}

The rapidly decreasing reserves of fossil fuels and environmental considerations are currently compelling researchers to discover efficient alternative energy sources. The renewable energy source (RES) is a widespread concept covering wind energy, solar energy, biomass, geothermal, and tidal wave energies which are believed to have the ability to tackle our dependency on fossil fuels. Extensive studies about electrical energy generation by using RESs have been performed by numerous research laboratories and scientists ( $\mathrm{Fu}$ et al., 2014; Jain \& Agarwal, 2008; Kabalci, Kabalci, \& Develi, 2012; Liserre, Sauter, \& Hung, 2010; Nehrir et al., 2011; Spagnuolo et al., 2010; Yu, Zhang, Xiao, \& Choudhury, 2011). At present, RESs are assumed as alternative energy sources to fuels, and they can be easily integrated into currently used grid infrastructures. Since an energy plant that is based on RES needs to permanently supply the electrical loads as well as the conventional grid, stan-

\footnotetext{
* Corresponding author.

E-mail address: develi@erciyes.edu.tr (I. Develi).

Peer Review under the responsibility of Universidad Nacional Autónoma de México.
}

dalone renewable energy plants are assisted with battery and energy storage systems.

Distributed energy sources (DES) are suggested as a solution to energy demands by interconnecting several different energy sources together. A robust microgrid structure that is constituted from various energy sources should be able to be easily connected to and disconnected from the conventional grid; this requirement means the integration capability of any additional source connection to the existing distributed generation system without requiring any system configuration (Spagnuolo et al., 2010; Yu et al., 2011). The monitoring and metering requirements of microgrid networks should be met as well as conducted in conventional grids. Although several measuring methods have been proposed such as wired or wireless, all the solutions are related to the smart grid concept that has been extensively researched (Gungor et al., 2011; Matanza, Alexandres, \& Rodríguez-Morcillo, 2014; Sung \& Hsu, 2013; Wen, Wang, Zhu, Li, \& Zhou, 2013; Yan, Qian, Sharif, \& Tipper, 2013). The smart grid should meet the remote sensing, communication, controlling, monitoring and analysis demands in a sustainable, secure and efficient way to manage the entire infrastructure. Smart grid applications are widely used in the phase measurement, advanced metering and remote monitoring 
of source and load amplitudes (Galli, Scaglione, \& Wang, 2010; Gungor et al., 2011; Kabalci et al., 2012; Matanza et al., 2014; Sung \& Hsu, 2013; Yan et al., 2013; Yu et al., 2011).

The type of communication medium preferred in smart grids is wired or wireless because of environmental and substructural factors. Wireless communication systems are standardized to wireless home area networks (HAN) and wireless wide area networks (WAN). Besides these standard networks, business area networks (BANs) and neighborhood area networks (NANs) have also been proposed by several researchers and alliances. The HAN standards are implemented and operated by ZigBee alliance and Wi-Fi alliance which include the global system for mobile communications (GSM), ZigBee, Wi-Fi, and the general packet radio service (GPRS), while the WAN standards cover WiMax that is based on IEEE 802.16 standard (Gungor et al., 2011; Yan et al., 2013; Yu et al., 2011). Although wireless communication seems to be the most convenient method to transfer information and control data, the required infrastructure greatly increases the installation costs. Furthermore, the destructive effects of the communication medium decrease the sustainability and security of data transfer in wireless communication. An alternative method to transmit measured data is to use electrical power lines as a transmission medium; this concept is defined as power line communication (PLC). PLC is considered as a promising technology in smart grids because of its eliminating the additional costs of wireless and other wire line communication methods (Galli et al., 2010), and owing to its high-speed data rates of up to 200 Megabits per second (Mbps). Moreover, PLC can be used in industrial, indoor, and outdoor applications thanks to its various communication bands that are called broadband (BB) and narrowband (NB) (Galli et al., 2010; Gungor et al., 2011; Yan et al., 2013). NB PLC systems operate below $500 \mathrm{kHz}$ band according to CENELEC, FCC or ARIB standards and generally utilize single carrier systems. On the other hand, BB PLC systems run between the band range of $1-30 \mathrm{MHz}$ and exploit multi carrier systems such as orthogonal frequency-division multiplexing (OFDM), and especially coded OFDM. Recently reported studies in the literature showed that the low-density parity-check (LDPC) code is the best solution for the channel coding process in PLC systems (Andreadou \& Pavlidou, 2010; Andreadou, Assimakopoulos, \& Pavlidou, 2007; Nakagawa, Umehara, Denno, \& Morihiro, 2005; Spencer, 2005; Wada, 2004). The authors in (Andreadou et al., 2007) aimed to compare LDPC code performance with Reed-Solomon and Convolutional codes over the PLC channels and they showed that LDPC codes perform better than other codes. In Nakagawa et al. (2005), the authors searched for a way to improve the decoding process of LDPC codes over PLC channels with impulsive noise. The performance of high rate and short-block LDPC codes in low bandwidth PLC systems is considered in Spencer (2005). The authors in Wada (2004) showed that the performance of LDPC codes is also better than that of Turbo codes in PLC channels. The performance of irregular quasi-cyclic (QC) LDPC codes over a statistical PLC channel model with highly impulsive noise is examined in Andreadou and Pavlidou (2010).
In previous studies (Develi \& Kabalci, 2014a; Kabalci, Develi, \& Kabalci, 2013), we have examined bit error rate (BER) performances of LDPC coded OFDM systems over Canete's PLC channel model and aimed to show superiority of LDPC codes among others in PLC channels. In Develi and Kabalci (2014b), effect of using different decoding schemes on the LDPC coded OFDM systems over indoor PLC channels was analyzed. In addition to these works, an image transmission system for smart grids was also proposed in (Develi, Kabalci, \& Basturk, 2014). Furthermore, a PLC channel model proposal that is based on practical channel measurements acquired from electrical networks in Turkey was reported in Develi, Kabalci, and Basturk (2015).

This paper presents investigation of the QC-LDPC coded OFDM system performances over a practical PLC channel in contrast to previous studies reported in the literature. To obtain a realistic PLC channel medium, long-term measurements were carried out in Nigde Vocational College of Technical Sciences, Turkey. The simulation results were obtained by varying the code rate, block length and iteration numbers of the QC-LDPC codes over the generated PLC channel. Furthermore, the simulations were not only completed by transmitting randomly generated data, but were also carried out for the transmission of different images such as lenna, cameraman and baboon which are widely used for performance evaluation and comparison in the literature. As a result, it was confirmed that the QC-LDPC coded OFDM system, in which performance was analyzed in a real PLC channel, can be utilized in smart grids for communication and remote monitoring purposes in a reliable way.

The rest of this paper is organized as follows: Section 2 describes the QC-LDPC codes, the OFDM system principles and system model. The PLC channel measurement system is explained in Section 3. Finally, the simulation results and conclusions are given in Sections 4 and 5, respectively.

\section{System model}

\section{1. $L D P C$ and $Q C-L D P C$ codes}

LDPC codes are robust error correcting codes and are a special type of linear block code (Gallager, 1963). A sparse matrix, called parity-check matrix $\mathbf{H}$, is exploited to define these codes. When an $(n, k)$ LDPC code is considered, $k$ denotes information bits and $n$ represents coded bits with an $r=k / n$ code rate. In addition, the dimensions of the parity-check matrix $\mathbf{H}$ are shown by $(n-k) \times n$. LDPC codes have some advantages, such as a simple coding process, parallel and iterative decoding operations and good performance when they are compared with Reed-Solomon, Convolutional or Turbo codes. Because of their high performance and low decoding complexity compared to other channel coding schemes, LDPC codes have been exploited in most modern communication systems such as DVB-S2/-T2/C2, 802.11n (Wi-Fi), 802.16e (WiMAX), IEEE802.3an (10Gbit Ethernet) and G.hn/G.9960.

QC-LDPC codes, which are a special type of LDPC code, offer a simple encoding process and better error correcting performance. These codes utilize the shifting method to decrease 
encoding complexity. In addition, they can be easily implemented because of their cyclic characteristic (Myung, Yang, \& Kim, 2005). A parity-check matrix that comprises sub-matrices called zero or circulant permutation matrices defines a binary QC-LDPC code. A permutation matrix $\mathbf{P}^{i}$ of size $L \times L$ is given with (1).

$\mathbf{P}^{i}=\left[\begin{array}{ccccc}0 & 1 & 0 & \ldots & 0 \\ 0 & 0 & 1 & \ldots & 0 \\ \vdots & \vdots & \vdots & \ddots & \vdots \\ 0 & 0 & 0 & \ldots & 1 \\ 1 & 0 & 0 & \ldots & 0\end{array}\right]$

It is important to note that the circulant permutation matrix $\mathbf{P}^{i}$ shifts the identity matrix $\mathbf{I} i$ times to the right as long as the $0 \leq i<L$ condition is true. Finally, the parity-check matrix $\mathbf{H}$ of size $m L \times n L$ is given as (Myung et al., 2005)

$\mathbf{H}=\left[\begin{array}{cccc}\mathbf{P}^{a_{11}} & \mathbf{P}^{a_{12}} & \cdots & \mathbf{P}^{a_{1 n}} \\ \mathbf{P}^{a_{21}} & \mathbf{P}^{a_{22}} & \ldots & \mathbf{P}^{a_{2 n}} \\ \vdots & \vdots & \vdots & \vdots \\ \mathbf{P}^{a_{m 1}} & \mathbf{P}^{a_{m 2}} & \cdots & \mathbf{P}^{a_{m n}}\end{array}\right]$

where $a_{i j} \in\{0,1, \ldots, L-1, \infty\}, 1 \leq i<m$ and $1 \leq j<n$.

On the other hand, the decoding process of the QC-LDPC codes can be performed by using soft- and hard-decision decoder schemes similar to LDPC codes (Chen, Dholakia, Eleftheriou, Fossorier, \& Hu, 2005; Gallager, 1963; Jiang, Peng, Song, Pan, \& Yang, 2009; Phakphisut, Supnithi, Sopon, \& Myint, 2011; Zhong, Xu, Xie, \& Zhang, 2007). Although hard decision decoders have the advantage of low complexity, they always exhibit lower performance than that of soft decision decoders. The most commonly used soft-decision decoder is the Belief Propagation (BP) decoder (Chen et al., 2005) that performs the decoding process in an iterative way; it is also utilized in this study for the decoding process.

\section{2. $Q C$-LDPC coded OFDM system design for monitoring energy systems}

Orthogonal frequency-division multiplexing (OFDM) (Chang, 1966) is a transmission method which, as the name implies, utilizes more than one orthogonal carrier. An OFDM system consists of both modulation and multiplexing infrastructures (Chang, 1966; Hwang, Yang, Wu, Li, \& Li, 2009; Wu \& Zou, 1995; Zou \& Wu, 1995). The most advantageous properties of the OFDM are bandwidth efficiency and its robust structure against channel fading. Inter Symbol Interference (ISI) has attracted the attention of researchers and caused it to be widely researched. The system can divide a frequency selective channel into several sub-channels with parallel fading properties that require proportionately simple processes for channel equalization. Several standards such as asymmetric digital subscriber lines (ADSL), very high-bit-rate digital subscriber lines (VDSL), digital television, radio broadcasting and wireless local area networks (WLAN) systems currently use OFDM systems.

A block diagram of the proposed system for monitoring smart grids through power line communication is given in Fig. 1. As can be seen from the figure, the communication infrastructure of the monitoring system is based on QC-LDPC coded OFDM modems. To show the efficiency of the monitoring system, the performance of the QC-LDPC coded OFDM modem is tested by using digital data and lenna, cameraman and baboon images with $256 \times 256$ pixels. As mentioned before, these images are selected since they are widely used for the comparison of system performance in the literature. The transmission of image processing by using the QC-LDPC coded OFDM system over a PLC channel can be summarized briefly as follows.

Firstly, the LDPC encoder involves properly adjusted image data as the input. Hence, the pixels of the pattern image are converted to 8-bit grayscale digital data before being applied to the encoder. The initial block of the transmitter, the LDPC encoder, executes the channel-coding process for message bits that are transferred to the modulation process to map the encoded data stream. Afterwards, pilot symbols are integrated into the modulated data in order to obtain a precise estimation on the receiver side. In the following step, the parallel data that are generated by being converted from serial data are transferred to the inverse fast Fourier transform (IFFT) process where OFDM signals are generated in the time domain. The ISI effect is eliminated by adding a guard interval to the OFDM signals by cyclic prefixes. The parallel data are converted to serial data that are prepared for application to practical PLC channels at the back-end of the cyclic prefix insertion process.

The receiver part of the entire system starts after the PLC channels. The received data are firstly converted to parallel type and then are applied to the fast Fourier transform (FFT) processing by removing the guard interval. The output of the FFT process is again converted to serial data and then channel estimation and pilot symbol removing processes are applied to the serial data in the frequency domain. The demodulation and LDPC decoding processes configure the last step of the receiver side. The overall performance of the system is detected by comparing the peak signal-to-noise ratio (PSNR) of the transmitted and received image. The PSNR method that is widely used to detect image quality involves the calculation of the mean square error (MSE) as seen in (3)

$M S E=\frac{1}{x \times y} \sum_{x=0}^{x-1} \sum_{y=0}^{y-1}\|S(x, y)-R(x, y)\|^{2}$

where $x \times y$ defines the image size, and $S$ and $R$ stand for the transmitted and received image pixel values in binary form, respectively. On the other hand, the PSNR value can be defined by using the MSE as given below

$P S N R=10 \log _{10}\left(\frac{M A X^{2}}{M S E}\right)$ 


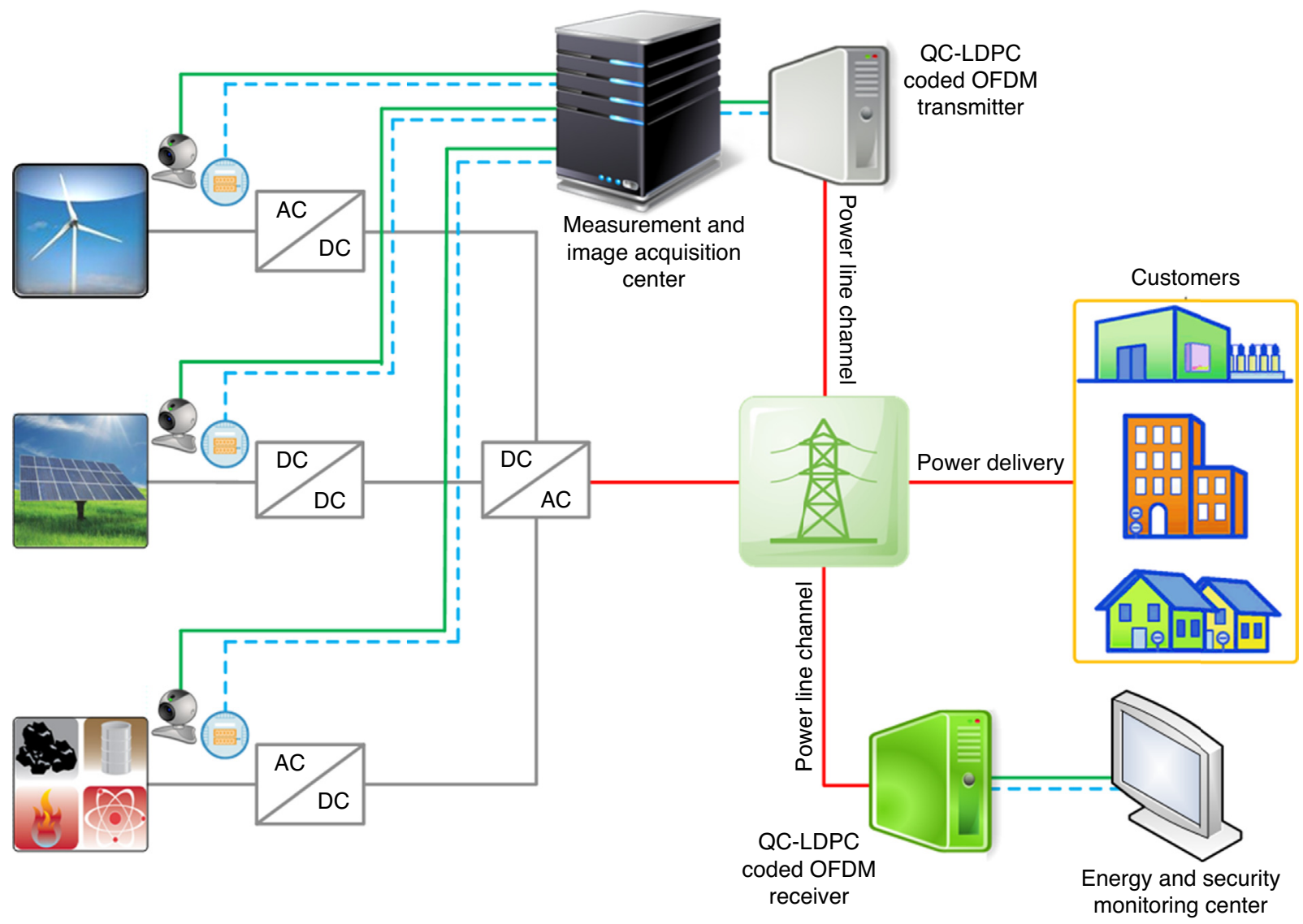

Fig. 1. Block diagram of the QC-LDPC coded OFDM systems for monitoring energy systems through power line communication.

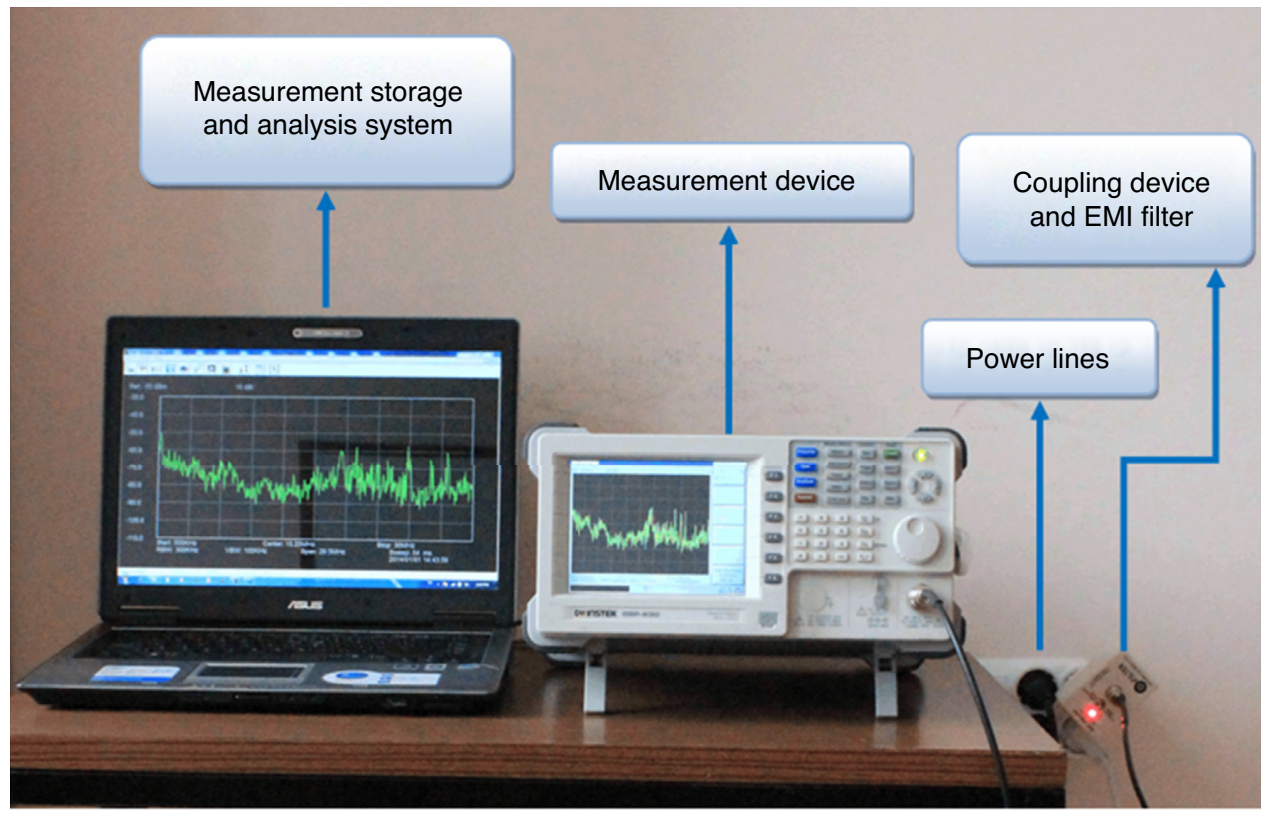

Fig. 2. Practical measurement system used to acquire characteristics of power lines. 


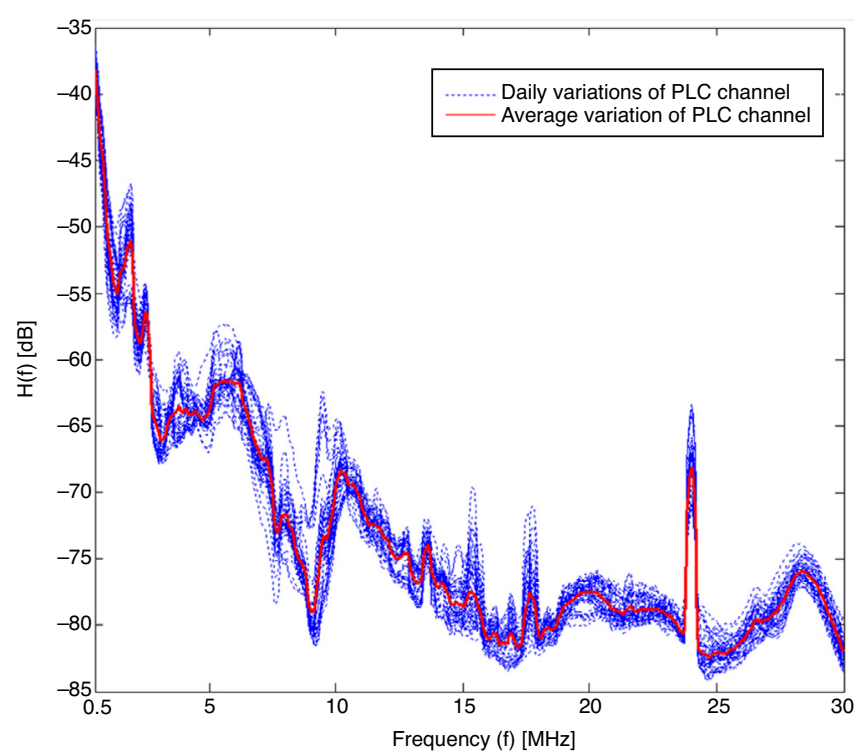

Fig. 3. PLC channel frequency responses measured in Nigde Vocational College of Technical Sciences, Turkey.

where $M A X$ depicts the maximum pixel value of the image. Since each of the pixels is converted to 8-bit data, the MAX value is set to 256 in this study.

\section{PLC channel measurement system}

The power line communication (PLC) measurement studies were carried out between $500 \mathrm{kHz}$ and $30 \mathrm{MHz}$ by installing the measurement testbed at Nigde Vocational College of Technical Sciences, Turkey. The practical measurement system used to acquire the characteristics of the power lines is illustrated in Fig. 2. As can be seen from the figure, secure measurement is performed owing to the coupling circuit that eliminates possible damages by electrically isolating the measurement system from the grid and severe loads connected to and disconnected from the grid. The measurement meter that is connected over coupling circuits provides reliability of measurement by obtaining the instant changes in the power line channel in terms of frequency domain.

Because the instant data acquisitions yield similar results to the scenario of an actual power line channel, the measurement processes are carried out for long lasting periods; for example, a period indicates $24 \mathrm{~h}$, and a long-term measurement takes two months. The instant measurements are transmitted to the computer and are saved as master files. Fig. 3 depicts the performed measurements and the average frequency response of the channel, $H(f)$, versus frequency $f$. When the measurements acquired from the practical power lines are analyzed, the daily variations of the PLC channel are observed within certain limits. The average value of the PLC channel, shown by the red curve in Fig. 3, is calculated and considered as a PLC channel medium in this study.
Table 1

Code rates and lengths of QC-LDPC codes employed in the simulations.

\begin{tabular}{lll}
\hline Code rates & \multicolumn{2}{c}{ Code lengths } \\
\cline { 2 - 3 } & Short-length codes & Long-length codes \\
\hline $1 / 4$ & $(900 \times 225)$ & $(1800 \times 450)$ \\
$1 / 3$ & $(900 \times 300)$ & $(1800 \times 600)$ \\
$1 / 2$ & $(900 \times 450)$ & $(1800 \times 900)$ \\
$2 / 3$ & $(900 \times 600)$ & $(1800 \times 1200)$ \\
$5 / 6$ & $(900 \times 750)$ & $(1800 \times 1500)$ \\
\hline
\end{tabular}

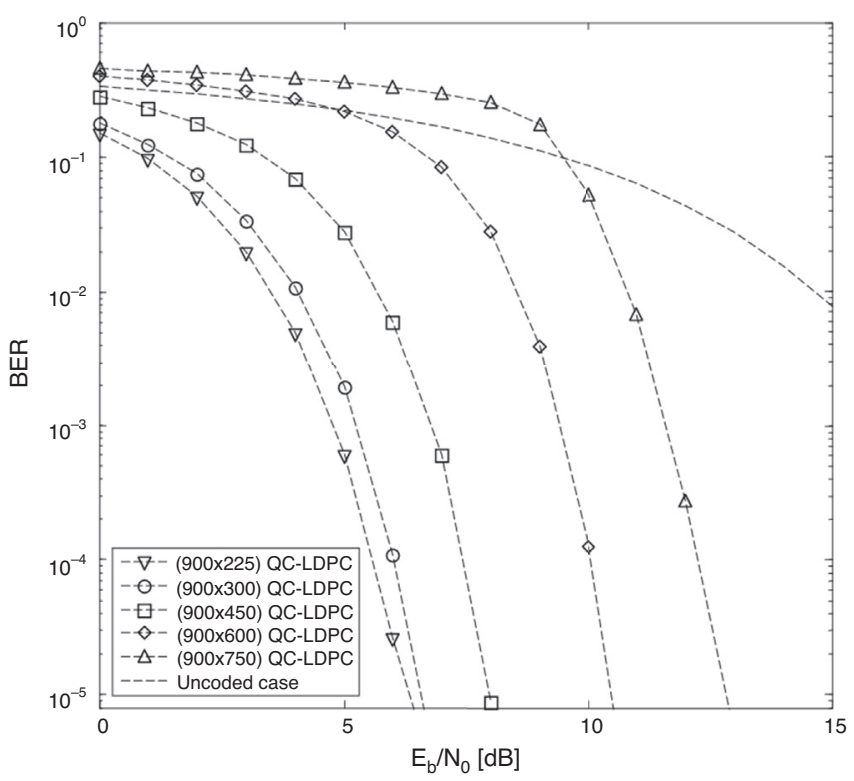

Fig. 4. BER performances of OFDM systems encoded with short-length QCLDPC codes over a realistic PLC channel.

\section{Results and discussion}

In this section, we investigate the performance of QC-LDPC coded OFDM systems over a PLC channel obtained with practical measurements in terms of data and image transmission. In order to perform a comprehensive analysis, ten differentlength QC-LDPC codes with various code rates were examined through computer simulations. Table 1 gives the code rates and code lengths utilized in this study. In addition, a BP decoder scheme is preferred on the receiver of the modeled communication system because of its good performance and the maximum iteration number of the decoder was set to 10 and 50 for the data and image transmission process, respectively. While the performance results for data transfer are presented in Figs. 4 and 5, the results for image transmission over a practical PLC channel are illustrated in Fig. 6.

Fig. 4 shows the Bit Error Rate (BER) performances of the OFDM system versus signal-to-noise ratio $\left(E_{b} / N_{0}\right)$ when the system is encoded by using short-length QC-LDPC codes. It is clearly seen from Fig. 4 that the BER performance of the OFDM system is highly increased when QC-LDPC codes are employed. When high-density codes are compared with the uncoded case, the improvement offered by the $(900,600)$ code is nearly $6.25 \mathrm{~dB}$ while the $(900,750)$ code provides $4 \mathrm{~dB}$ better performance for 


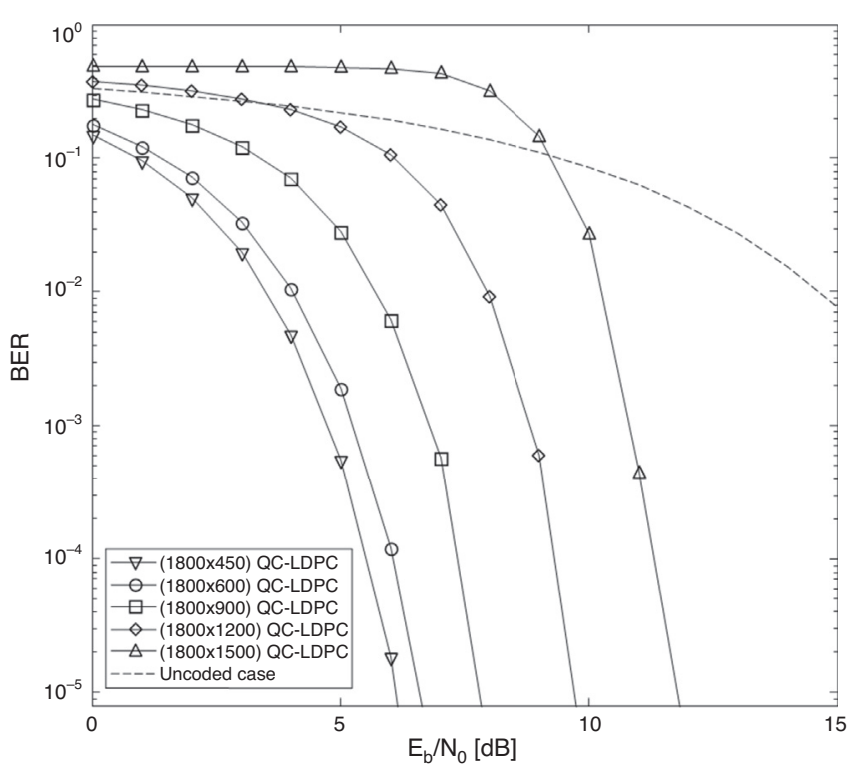

Fig. 5. BER performance results of OFDM systems over a realistic PLC channel with long-length QC-LDPC codes. a BER level of $10^{-2}$. In addition, the QC-LDPC code with a $1 / 4$ rate provides approximately $11.4 \mathrm{~dB}$ improvement at the BER level of $10^{-2}$.

The BER performance results of the QC-LDPC coded OFDM system obtained by using long-length codes are illustrated in Fig. 5. It is observed that, when the uncoded system is considered, about $15 \mathrm{~dB}$ is needed for a BER value of $10^{-2}$. However, the similar performance with $2 / 3$ and $5 / 6$ rate QCLDPC codes are presented at $E_{b} / N_{0} \approx 8.1 \mathrm{~dB}$ and $E_{b} / N_{0} \approx$ $10.4 \mathrm{~dB}$, respectively.

In cases where the $1 / 3$ and $1 / 2$ rate $\mathrm{QC}$-LDPC codes are utilized instead of high-density codes, the achieved gains with respect to the uncoded case by these codes are $10.75 \mathrm{~dB}$ and $9 \mathrm{~dB}$ at a BER level of $10^{-2}$, respectively. In addition, when the $1 / 4$ rate code is used, about $6 \mathrm{~dB}$ is required for a BER value of $10^{-5}$. Finally, we can see from Figs. 4 and 5 that long-length QC-LDPC codes provide nearly $0.75 \mathrm{~dB}$ better performance than that of the short-length codes in high rates.

Fig. 6 indicates the image transmission results for the QCLDPC coded OFDM system with the $(1800,600)$ code over the PLC channel. When the coded system results shown in Fig. 6a-c are compared with the uncoded cases depicted in Fig. 6d-f, the a

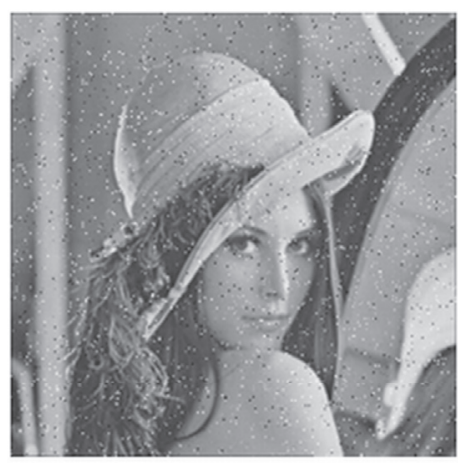

$\mathrm{SNR}=4 \mathrm{~dB}$

$P S N R=23.03 \mathrm{~dB}$

d

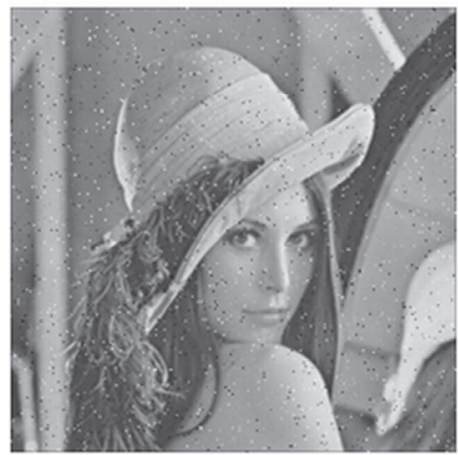

$\mathrm{SNR}=26 \mathrm{~dB}$

$P S N R=24.75 \mathrm{~dB}$ b

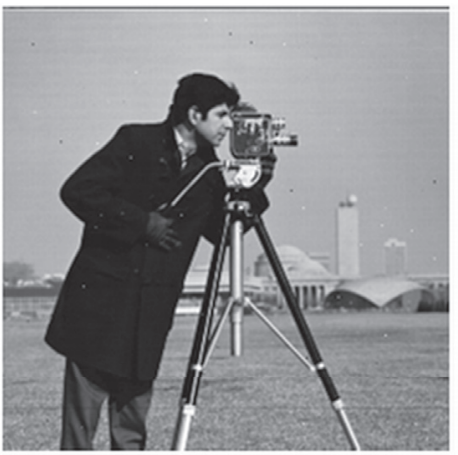

$\mathrm{SNR}=6 \mathrm{~dB}$

$P S N R=39.95 \mathrm{~dB}$

e

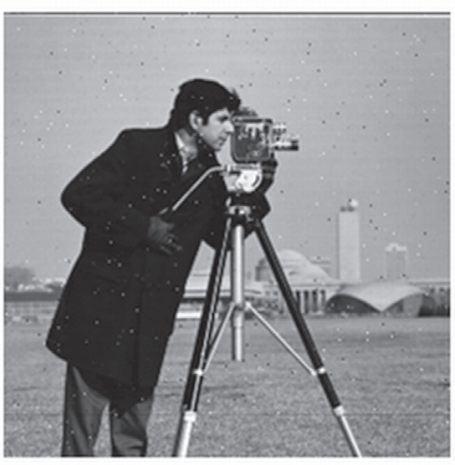

$\mathrm{SNR}=28 \mathrm{~dB}$

$\mathrm{PSNR}=31.86 \mathrm{~dB}$
C

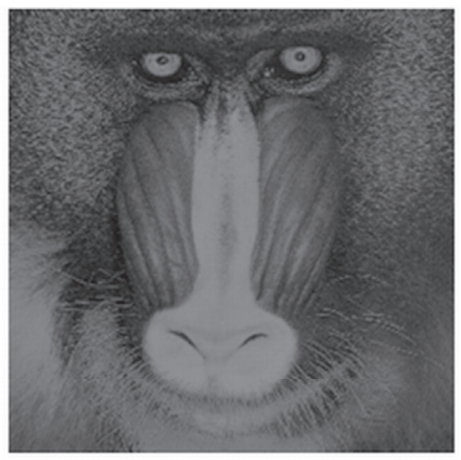

$\mathrm{SNR}=8 \mathrm{~dB}$

$P S N R=61.07 \mathrm{~dB}$

f

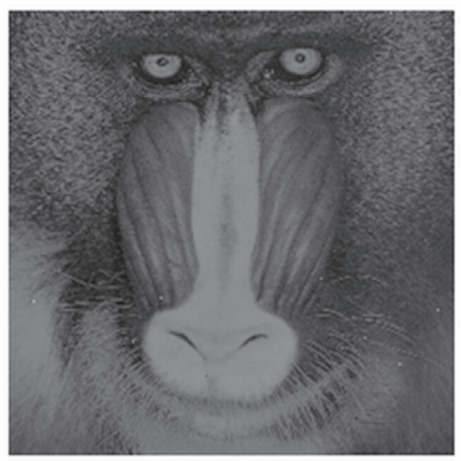

$\mathrm{SNR}=30 \mathrm{~dB}$

$P S N R=43.21 \mathrm{~dB}$

Fig. 6. Comparison of uncoded and QC-LDPC coded OFDM systems for different images in PLC channels ((a), (b) and (c) present QC-LDPC code performances; (d), (e) and (f) show uncoded cases). 
PSNR value of $24.75 \mathrm{~dB}$ is obtained at a $26 \mathrm{~dB}$ SNR value in the uncoded OFDM system, while nearly the same PSNR performance can be achieved at a lower SNR value such as $4 \mathrm{~dB}$ in the coded system for the lenna image. In the event of the cameraman image results for the same QC-LDPC code presented in Fig. 6 are analyzed, it is shown that about $28 \mathrm{~dB}$ SNR is needed for a medium quality image transmission with a 31.86 PSNR value in the uncoded system. However, better performance is provided at the $6 \mathrm{~dB}$ SNR value in the coded OFDM system. When the transmission results for the baboon images using $1 / 3$ rate and long-length QC-LDPC codes are considered, it is clearly seen from Fig. 6 that very high quality image transmission can be obtained at the SNR value of $8 \mathrm{~dB}$ and an extremely high PSNR value of $61.07 \mathrm{~dB}$ owing to the coded OFDM system.

As a final remark, all of the simulation results show that QC-LDPC codes provide significant improvement in terms of data and image transmission over the practical PLC channel. In addition, the results obtained confirm that the QC-LDPC coded OFDM system can be utilized in smart grids for remote monitoring energy systems.

\section{Conclusion}

This paper investigated the performance of QC-LDPC coded OFDM systems for both data and image transmission using practical PLC channel conditions. The long-term PLC channel measurements were carried out to obtain an actual channel model. In order to realize a comprehensive analysis, the simulation studies examined both different code lengths and code rates. All of the simulation results achieved over the actual PLC channel show that the QC-LDPC codes ensure considerable improvement in terms of data and image transmission performance. The results of this study also emphasize that the QC-LDPC coded OFDM system tested in an actual PLC channel can be reliably utilized in smart grids for communication and remote monitoring purposes.

\section{Conflicts of interest}

The authors have no conflicts of interest to declare.

\section{Acknowledgements}

This work was supported by the Scientific and Technological Research Council of Turkey (TUBITAK) under grant 113E425 and by the Research Fund of Erciyes University under grant FBD-12-3986.

\section{References}

Andreadou, N., \& Pavlidou, F. N. (2010). Mitigation of impulsive noise effect on the PLC channel with QC-LDPC codes as the outer coding scheme. IEEE Transactions on Power Delivery, 25(3), 1440-1449.

Andreadou, N., Assimakopoulos, C., \& Pavlidou, F. N. (2007). Performance evaluation of LDPC codes on PLC channel compared to other coding schemes. In Power line communications and its applications symposium, 2007, IEEE (pp. 296-301).
Chang, R. W. (1966). Synthesis of band-limited orthogonal signals for multichannel data transmission. The Bell System Technical Journal, 45(10), 1775-1796.

Chen, J., Dholakia, A., Eleftheriou, E., Fossorier, M. P. C., \& Hu, X. Y. (2005). Reduced-complexity decoding of LDPC codes. IEEE Transactions on Communications, 53(8), 1288-1299.

Develi, I., \& Kabalci, Y. (2014a). Highly reliable LDPC coded data transfer in home networks by using Canete's PLC channel model. International Journal of Electrical Power \& Energy Systems, 62, 912-918.

Develi, I., \& Kabalci, Y. (2014b). Analysis of the use of different decoding schemes in LDPC coded OFDM systems over indoor PLC channels. Elektronika Ir Elektrotechnika, 20(10), 76-79.

Develi, I., Kabalci, Y., \& Basturk, A. (2014). Performance of LDPC coded image transmission over realistic PLC channels for smart grid applications. International Journal of Electrical Power \& Energy Systems, 62, 549-555.

Develi, I., Kabalci, Y., \& Basturk, A. (2015). Artificial bee colony optimization for modelling of indoor PLC channels: A case study from Turkey. Electric Power Systems Research, 127, 73-79.

Fu, Q., Nasiri, A., Bhavaraju, V., Solanki, A., Abdallah, T., \& David, C. Y. (2014). Transition management of microgrids with high penetration of renewable energy. IEEE Transactions on Smart Grid, 5(2), 539-549.

Gallager, R. G. (1963). Low-density parity-check codes. Cambridge, MA: MIT Press.

Galli, S., Scaglione, A., \& Wang, Z. (2010). Power line communications and the smart grid. In In Smart grid communications conference, 2010, IEEE (pp. 303-308).

Gungor, V. C., Sahin, D., Kocak, T., Ergut, S., Buccella, C., Cecati, C., et al. (2011). Smart grid technologies: Communication technologies and standards. IEEE Transactions on Industrial Informatics, 7(4), 529-539.

Hwang, T., Yang, C., Wu, G., Li, S., \& Li, G. Y. (2009). OFDM and its wireless applications: A survey. IEEE Transactions on Vehicular Technology, 58(4), 1673-1694

Jain, S., \& Agarwal, V. (2008). An integrated hybrid power supply for distributed generation applications fed by nonconventional energy sources. IEEE Transactions on Energy Conversion, 23(2), 622-631.

Jiang, N., Peng, K., Song, J., Pan, C., \& Yang, Z. (2009). Highthroughput QC-LDPC decoders. IEEE Transactions on Broadcasting, 55(2), 251-259.

Kabalci, E., Kabalci, Y., \& Develi, I. (2012). Modelling and analysis of a power line communication system with QPSK modem for renewable smart grids. International Journal of Electrical Power \& Energy Systems, 34, 19-28.

Kabalci, Y., Develi, I., \& Kabalci, E. (2013). LDPC coded OFDM systems over broadband indoor power line channels: A performance analysis. In Powereng conference, 2013. IEEE (pp. 1581-1585).

Liserre, M., Sauter, T., \& Hung, J. Y. (2010). Future energy systems: Integrating renewable energy sources into the smart power grid through industrial electronics. IEEE Industrial Electronics Magazine, 4(1), 18-37.

Matanza, J., Alexandres, S., \& Rodríguez-Morcillo, C. (2014). Advanced metering infrastructure performance using European low-voltage power line communication networks. IET Communications, 8(7), 1041-1047.

Myung, S., Yang, K., \& Kim, J. (2005). Quasi-cyclic LDPC codes for fast encoding. IEEE Transactions on Information Theory, 51(8), 2894-2901.

Nakagawa, H., Umehara, D., Denno, S., \& Morihiro, Y. (2005). A decoding for low density parity check codes over impulsive noise channels. In Power line communications and its applications symposium, 2005. IEEE (pp. 85-89).

Nehrir, M. H., Wang, C., Strunz, K., Aki, H., Ramakumar, R., Bing, J., et al. (2011). A review of hybrid renewable/alternative energy systems for electric power generation: Configurations, control, and applications. IEEE Transactions on Sustainable Energy, 2(4), 392-403.

Nakagawa, H., Umehara, D., Denno, S., \& Morihiro, Y. (2005). A decoding for low density parity check codes over impulsive noise channels. In Power line communications and its applications symposium, 2005. IEEE (pp. 85-89).

Nehrir, M. H., Wang, C., Strunz, K., Aki, H., Ramakumar, R., Bing, J., et al. (2011). A review of hybrid renewable/alternative energy systems for electric power generation: Configurations, control, and applications. IEEE Transactions on Sustainable Energy, 2(4), 392-403. 
Phakphisut, W., Supnithi, P., Sopon, T., \& Myint, L. M. M. (2011). Serial belief propagation for the high-rate LDPC decoders and performances in the bit patterned media systems with media noise. IEEE Transactions on Magnetics, 47(10), 3562-3565.

Spagnuolo, G., Petrone, G., Vasconcelos, S., Cecati, A. C., Friis-Madsen, E., Gubia, E., et al. (2010). Renewable energy operation and conversion schemes: A summary of discussions during the seminar on renewable energy systems. IEEE Industrial Electronics Magazine, 4(1), 38-51.

Spencer, Q. H. (2005). Short-block LDPC codes for a low-frequency power-line communications system. In Power line communications and its applications symposium, 2005. IEEE (pp. 95-99)

Sung, W. T., \& Hsu, C. C. (2013). Intelligent environment monitoring system based on innovative integration technology via programmable system on chip platform and ZigBee network. IET Communications, 7(16), 1789-1801.

Wada, T. (2004). A study on performance of LDPC codes on power line communications. In Communications conference, 2004. IEEE (pp. 109-113).
Wen, H., Wang, Y., Zhu, X., Li, J., \& Zhou, L. (2013). Physical layer assist authentication technique for smart meter system. IET Communications, 7(3), 189-197.

Wu, Y., \& Zou, W. Y. (1995). Orthogonal frequency division multiplexing: A multi-carrier modulation scheme. IEEE Transactions on Consumer Electronics, 41(3), 392-399.

Yan, Y., Qian, Y., Sharif, H., \& Tipper, D. (2013). A survey on smart grid communication infrastructures: Motivations, requirements and challenges. IEEE Communications Surveys \& Tutorials, 15(1), 5-20.

Yu, F. R., Zhang, P., Xiao, W., \& Choudhury, P. (2011). Communication systems for grid integration of renewable energy resources. IEEE Network, 25, 22-29.

Zhong, H., Xu, W., Xie, N., \& Zhang, T. (2007). Area-efficient min-sum decoder design for high-rate quasi-cyclic low-density parity-check codes in magnetic recording. IEEE Transactions on Magnetics, 43, 4117-4122.

Zou, W. Y., \& Wu, Y. (1995). COFDM: An overview. IEEE Transactions on Broadcasting, 41(1), 1-8. 